\title{
SAMPLING STRATEGIES FOR METAMODEL ENRICHMENT AND AUTOMOTIVE FAN OPTIMIZATION
}

\author{
M. Henner ${ }^{1}$ - B. Demory ${ }^{1-}$ T. Gonon ${ }^{1}-$ C. Helbert $^{2}$ \\ ${ }^{1}$ VALEO Thermal Systems - France \\ ${ }^{2}$ Ecole Centrale de Lyon - Institut Camille Jordan - UMR CNRS 5208 - Ecully, France
}

\begin{abstract}
The strategy aimed at reducing the development time for a fan, which is oriented through the use of large meta-models which can be re-used and enriched along time. Efforts have been brought in the parameterization of the geometry and in the simulation process which provides pressure rise, torque and consequently efficiency.

Numerical Designs of Experiments (DoE) are then conducted in an 11 factor problem to fill the space and to build a first kriging model. This latter assumes that the output is a gaussian field for which parameters are computed by maximum likelihood estimation based on the numerical runs. Uncertainties associated with any predicted values can then be assessed using the variance, and two small validation plans are used to measure statistically the errors.

The variance given by the model is further used to map the areas in the domain which would need additional sampling. Then, two strategies are tested to select the most relevant sampling points, the first one being to reduce the range of the parameter variation, and the other one being to select them according to turbomachine design rules which would have dismiss some factor combinations. These two methods for sequential enrichment of the response are then compared and can even be combined with a trend given on the pressure rise. Answers from the kriging models are then assessed again in terms of statistical errors. These strategies will be described in the proposed paper through model comparison and optimization results.
\end{abstract}

\section{KEYWORDS}

Fan system - Optimization - Kriging - Sampling - Design of Experiment

\section{NOMENCLATURE}

DoE Design of experiment

RMSE Root Mean Square of the prediction Error of the metamodel on a test sample

\section{LIST OF SYMBOLS}

A' transpose of $\mathrm{A}$, with A a matrix

$x=\left(x_{1}, \ldots, x_{11}\right) \quad$ vector of design variables (input variables), called point

$\mathrm{Y}(\mathrm{x}) \quad$ gaussian process, function of the design variables, used by the kriging model for the output

$\mathrm{m} \quad$ mean of the gaussian process $\mathrm{Y}$

$\mathrm{Z}(\mathrm{x}) \quad$ gaussian process with zero mean part of the writing of $\mathrm{Y}$ 


\begin{tabular}{|c|c|}
\hline $\mathrm{k}\left(\mathrm{x}, \mathrm{x}^{\prime}\right)$ & covariance kernel of Z \\
\hline$\sigma^{2}$ & variance of $\mathrm{Z}$ \\
\hline$\theta$ & range of $\mathrm{Z}$ \\
\hline$r_{\theta}\left(x, x^{\prime}\right)$ & correlation kernel of Z \\
\hline $\mathcal{L}\left(\mathrm{m}, \sigma^{2}, \theta\right)$ & likelihood function of $m, \sigma^{\wedge} 2$, and $\theta$ \\
\hline$x^{(i)}$ & $\mathrm{i}^{\text {th }}$ training point, or $\mathrm{i}^{\text {th }}$ test point \\
\hline $\mathrm{X}$ & matrix of all training points, also called design matrix, its lines are the $x^{\wedge}((i))$ \\
\hline $\mathrm{y}$ & vector of output values associated with training points, or with test points \\
\hline $\mathrm{R}$ & correlation matrix of the training points \\
\hline $\mathrm{r}(\mathrm{x})$ & correlation vector between $\mathrm{x}$ and the training points \\
\hline$\alpha_{\mathrm{i}}$ & coefficient \\
\hline $\mathrm{h}_{\mathrm{i}}\left(\mathrm{x}_{\mathrm{i}}\right)$ & usual function applied to a design variable \\
\hline $\begin{array}{l}\text { RMSE } \\
\mathrm{N}\end{array}$ & $\begin{array}{l}\text { Root Mean Square of the prediction Error of the metamodel on a test sample } \\
\text { number of test points }\end{array}$ \\
\hline$n$ & number of training points \\
\hline$f(x)$ & computer code value \\
\hline$\widehat{m}(x)$ & kriging prediction, also called Kriging mean \\
\hline$\hat{\sigma}^{2}(x)$ & variance of kriging prediction, also called kriging variance \\
\hline
\end{tabular}

\section{INTRODUCTION}

Specifications in automotive applications can cover a wide range of operating points since design, architecture and engine power are very different from one vehicle model to the other. Each new development for a fan involves therefore a new optimization process to reach nominal pressure rise and torque. Typical pressure and efficiency curves versus flow rate are presented in figure 1 with indications on operating points.

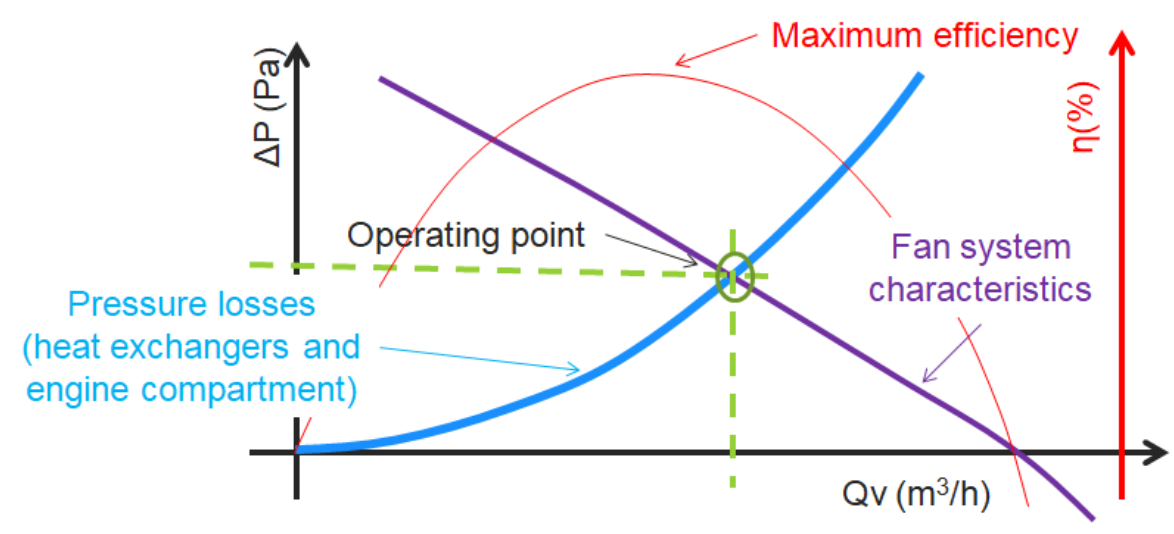

Figure 1: pressure and efficiency curves with operating points

Optimization is therefore perfectly adapted, and the current strategies aimed at reducing the development time for the fan, and are then oriented through the use of large meta-models which can be re-used and enriched along time. Investigations done on the current 11 parameters DoE are aimed to prepare a methodology for large dimensions, i.e. starting from 30 and going beyond, possibly up to 60. Accuracy must not be compromised neither by the space dimension (given by the number of parameters), nor by the widest range of possible variations. However, each simulation run has a non-neglectable cost which is not compatible with an intense sampling campaign. In the present case, 11 design variables have been selected and the full factorial plan would yield $2^{11}$ simulations, which is of course simply not possible. 
Some strategies are then investigated in order to enrich initial Design of Experiment (DoE) which are classically based on a statistical approach uncorrelated from the studied physics. The question is to find a proper way to select the most relevant points which would improve the accuracy. However, one can object that the accuracy is only of interest for some limited set of parameters, when geometries are conforming to turbomachine laws. Therefore, in the context of a limited simulation budget, should the enrichment be based on a purely statistical approach, or is it worthy to use geometrical rules which exclude any sampling on a priori non interesting areas?

\section{OBJECTIVES}

The objectives of the current work are to investigate a sequential strategy for fan optimization, in the context of a limited simulation budget, which uses at first a meta-model from a kriging method, and secondly a genetic algorithm for optima research. Such methods have been intensively used and presented for low speed fans, for instance by Bamberger [1] or Verstraete [2].

Several strategies for the selection of potential additional runs are proposed and compared between them. Performances of these methods are assessed through different criteria measured on each surrogate model, which are given by global or local variances, by discrepancies with numerical results from either a global or a local validation plan, and by comparing the optimized final designs.

Geometrical parameterization of the fans and simulation processes are explained in the next paragraph, followed by some theoretical information on the kriging method which has been used. Sequential plans and methods for comparison are then presented before giving results and commenting on their respective efficiencies in the conclusion.

\section{PARAMETERIZATION AND SIMULATIONS}

\section{Fan parameterization}

A typical automotive fan is presented in figure 2. Several characteristics are frozen to standard values, and the selected parameters for optimization are the chord lengths and the stagger angles for five different radius. These ten geometrical factors are then completed by the flow rate which is imposed in the numerical experiment.

DoE must have independent parameters and ensure that all their combinations are feasible in the ranges of variation, meaning that no effect between them would prevent any geometry to be tested. However, it can lead to the creation of designs which can be said at least unconventional, or at worst opposite to the rule of turbomachine design. One can appreciate in figure 3 , as an example a surprising resulting geometry on which the DoE can ask an evaluation of performances. In such cases, the heckled surfaces lead to chaotic flow patterns, yielding poor fan efficiencies and difficult numerical convergences (and consequently "signal” noise during the creation of meta-models). At a first thought, they are not representing the most interesting sampling points.

\section{Numerical modeling}

As presented in [3], performance predictions for the DoE are obtained, thanks to RANS simulations with the commercial code $\mathrm{CCM}+$, using polyhedra meshes and an accurate description of the test rig (figure 4). The simulation process takes one hour of meshing (a new mesh is produced for each geometry), and requires between 4 to 8 wall clock time with 196 CPU (depending on the convergence). Mesh parameters are kept constant from one geometry to the other in order to minimize discrepancies. An accuracy assessment is provided in [3], and allow us to determine the mesh size above which variations remain below $1 \%$. About 20 millions polyhedra are used, mainly 
concentrated in the rotating domain (15 millions). The motion of the fan in the steady state run is modeled through multiple frames of references. Turbulence is modeled with the classical 2 equations model k- $\omega$ SST from Menter, and a two layers model is used to predict accurately the boundary layer on the walls. The layers of cell extruded from the surface mesh yield to an average wall $\mathrm{y}+$ close to 1 on the fan blades. The flow rate is imposed at the inlet plenum of the test rig, whereas an atmospheric condition is imposed in the far field.

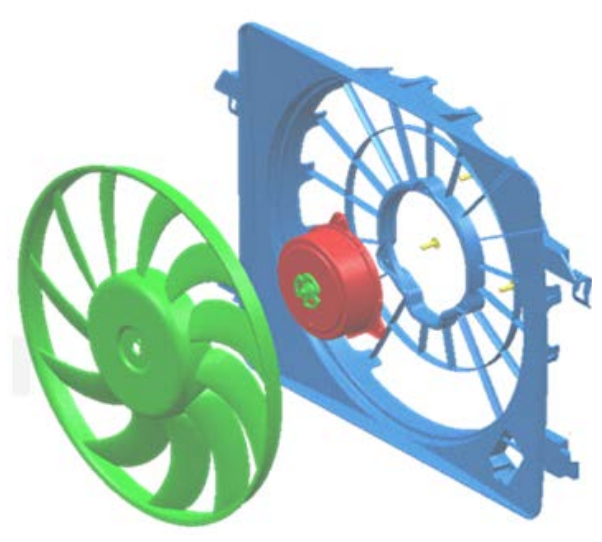

Figure 2: typical automotive fan
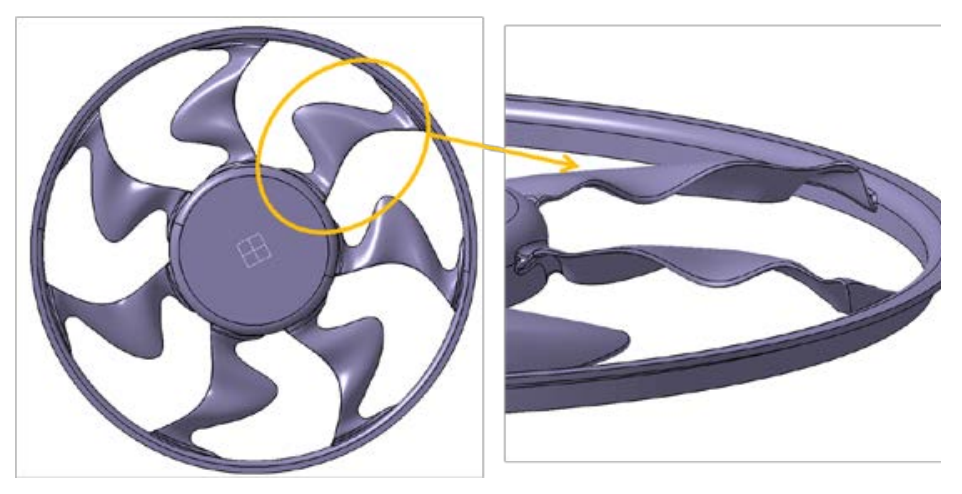

Figure 3: example of a surprising geometry

It allows us to monitor the performances with the pressure rise between an inlet and outlet (static to static) and the torque exerted by the fluid on the fan. Convergence is considered to be achieved when these values remain stable over a sufficient period of time, in addition to residues below $10^{-4}$. Selected output responses are then the pressure rise $(\Delta \mathrm{P}$ in $\mathrm{Pa})$, the torque $(\mathrm{T}$ in N.m) and the global efficiency (\%) deduced from the formula $\eta=\left(\Delta \mathrm{P}^{*} \mathrm{Q}\right) /\left(\mathrm{T}^{*} \Omega\right)$, $\mathrm{Q}$ being the flow rate $(\mathrm{m} 3 / \mathrm{s})$ and $\Omega$ the rotational speed ( $\mathrm{rad} / \mathrm{s})$.

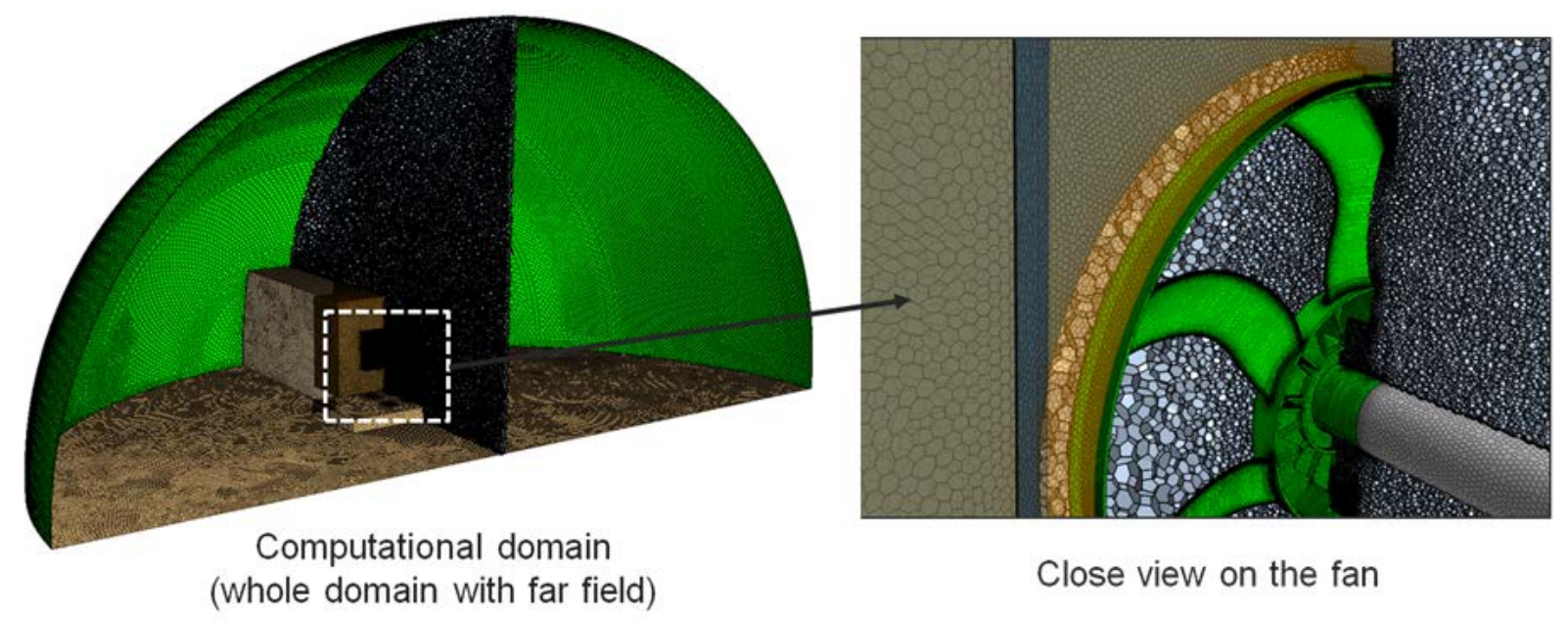

Figure 4: Simulation domain 


\section{DOE AND KRIGING METHOD}

\section{DOE's building [4]}

All factors have been dimensionalized in the range [-1, 1], the set of data with 11 factors at zero being the center of the domain. An Orthogonal Latin Hypercube (OLH) plan has been selected for the initial sampling and the training of the meta-model. It is composed of 127 points that have been selected in the $[-1,1]^{11}$ domain, according to the theory which imposes at first that each point must have a different value for each direction (a direction corresponding to an input variable), and secondly that the columns of the design matrix are orthogonal (one column contains the observed values of one design variable).

Two other Latin Hypercube Samples (LHS) designs (which were optimized for discrepancy) of 25 points each have been established for testing the meta-models trained from the 127 runs (and the points for enrichment if any). The first one is set in $[-1,1]^{11}$, and the second one in $[-0.5,0.5]^{11}$. For this later it means that all factors are limited in the range [-0.5, 0.5], i.e. only a central part of the domain is considered

\section{Kriging meta-model building [5]}

The kriging meta-model models the computer code, $\mathrm{f}$, as a realization of a random field $\{\mathrm{Y}(\mathrm{x}), \mathrm{x}$ in $\left.[-1,1]^{11}\right\}$. Each component of $\mathrm{x}$ is a particular input variable of the simulator. This random field is supposed to be such that:

$$
Y(x)=m+Z(x)
$$

where $m$ is a scalar constant, called trend, and $Z$ is a gaussian field with zero mean and a covariance function of the type :

$$
k\left(x, x^{\prime}\right)=\sigma^{2} r_{\theta}\left(x, x^{\prime}\right)
$$

$r_{\theta}$ is called the correlation function. The one used in this work is a tensor product of the Matern $\frac{5}{2}$ kernel :

$$
r_{\theta}\left(x, x^{\prime}\right)=\prod_{\mathrm{k}=1}^{11}\left(1+\frac{\sqrt{5}\left|\mathrm{x}_{\mathrm{k}}-\mathrm{x}_{\mathrm{k}}^{\prime}\right|}{\theta_{\mathrm{k}}}+\frac{5\left|\mathrm{x}_{\mathrm{k}}-\mathrm{x}_{\mathrm{k}}^{\prime}\right|}{3 \theta_{\mathrm{k}}^{2}}\right) \exp \left(-\frac{\sqrt{5}\left|\mathrm{x}_{\mathrm{k}}-\mathrm{x}_{\mathrm{k}}^{\prime}\right|}{\theta_{\mathrm{k}}}\right)
$$

The Matern $\frac{5}{2}$ kernel is the most commonly used because it is twice differentiable and it is the most realistic with respect to the actual simulation outputs. Other properties of gaussian kernel or exponential kernel are detailed for instance in [6].

The three parameters $m, \sigma^{2}$, and $\theta$ are determined by maximizing the likelihood function. $\theta$ is a vector of 11 components aimed to take into account of the anisotropy of the model. If we consider the set of training observations $y$, the likelihood function is:

$$
\mathcal{L}\left(m, \sigma^{2}, \theta\right)=\mathbb{P}\left(Y=y \mid m, \sigma^{2}, \theta\right)=\left(\frac{1}{2 \pi \sigma^{2}}\right)^{\frac{\mathrm{n}}{2}}\left(\frac{1}{\operatorname{det}(R)}\right)^{\frac{1}{2}} \exp \left(-\frac{\left(\mathrm{y}-\mathrm{m} 1_{\mathrm{n}}\right)^{\prime} R^{-1}\left(y-m 1_{n}\right)}{2 \sigma^{2}}\right)
$$


where $R=\left(r_{\theta}\left(x^{(i)}, x^{(j)}\right)\right)$ is the correlation matrix on the design points $x^{(1)}, \ldots, x^{(n)}$. The matrix of the observations (whose lines are the $x^{(i)}$ ) is noted $X$, and is called design matrix. The estimation of $m$ is different from the least square estimation. The least square estimation of $m$ would be given by the formula: $\frac{y^{(1)}+\cdots+y^{(n)}}{n}=\frac{\mathbf{1}_{n}^{\prime} y}{\mathbf{1}_{n}^{\prime} \mathbf{1}_{n}}$. However, the likelihood estimation of $m$ is given by the formula: $\frac{1_{n}^{\prime} R^{-1} y}{1_{n}^{\prime} R^{-1} 1_{n}}$. The least square estimation is a particular case of the maximum likelihood estimation when the correlation matrix $R$ is equal to the identity matrix.

Given that, the prediction is:

$$
\hat{y}(x)=E[Y(x) \mid Y(X)=y]=m+r^{\prime}(x) R^{-1}\left(y-m 1_{n}\right)
$$

where $r(x)=\left(r_{\theta}\left(x, x^{(i)}\right)\right)_{i}$ is the vector containing the correlation between $\mathrm{Y}(\mathrm{x})$ and $Y\left(x^{(1)}\right), \ldots, Y\left(x^{(n)}\right)$.

A kriging variance is also associated to each point of the input domain. It measures the uncertainty of the prediction at this point. The kriging variance is given by:

$$
\hat{\sigma}^{2}(x)=\sigma^{2}-r^{\prime}(x) R^{-1} r(x)
$$

Finally, if the main effects of the design variables $x_{k}$ are near known functions $h_{k}\left(x_{k}\right)$, this information can be added to the model by using a trend equal to a linear combination of the main effects rather than a constant trend. The prediction becomes:

$\hat{y}(x)=m+\sum_{k=1}^{n} \alpha_{k} h_{k}\left(x_{k}\right)+r^{\prime}(x) R^{-1}(y-F \alpha)$

where $F=\left(1_{n} H\right), H=\left(h_{k}\left(x_{k}^{(l)}\right)\right)_{l \in\{1, \ldots, n\}, k \in\{1, \ldots, 11\}}$, and $\alpha=\left(m \alpha_{1} \ldots \alpha_{n}\right)^{\prime}$.

The vector $\alpha$ is estimated by maximum likelihood. It is computed by the formula : $\left(F^{\prime} R^{-1} F\right)^{-1} F^{\prime} R^{-1} y$. The least square estimation would be : $\left(F^{\prime} F\right)^{-1} F^{\prime} y$. Again, the least square estimation is a particular case of the maximum likelihood estimation when the correlation matrix $R$ is equal to the identity matrix. model.

The goal is to improve the accuracy by adding global information on the "shape" of the meta-

\section{SAMPLING METHODS}

A previous approach studied by Ribaud [7] consists of adding points in the area of interest, extracting them from the Pareto front and selecting them according to a robustness criterion. This method is relevant for a known optimization problem (i.e. when the process can be guided in a limited area of the solution domain), but has no benefit for the global improvement of the metamodel. One proposal from Zhang [8] consists of, in using a small training sample (for example $\mathrm{OLH}$ ) and then in adding points. His method proved to be very fast because he could use a cokriging technique that drew additional information from the first and second derivatives. Of course, the great difficulty there is to have access to these values, and it implies either to derive the NavierStokes equations [9][10] or to assess the derivative with a differentiation method [11]. 


\section{Enrichment in a restricted hypercube}

The first trial consists of selecting one round of the 10 points according to the variance criterion introduced in the equation (6), the objective being to find the points with the maximum variance, which can help to improve the model globally by filling the most uncertain areas. The corners (factors equal to 1 or -1 ) are systematically proposed (figure 5), since they are in the extrapolation zone where the variance becomes large. Unfortunately, it would be hopeless to try any global improvement by selecting the $2^{11}$ corners. Alternatively, adding information in the center could be of interest since it would help to improve the model in the 11 directions. By limiting the selection in the $[-0.5,0.5]^{11}$ domain, it is observed that the first point is very close to the center (red point in figure 6), and then the other points are in the corners of the limited domain.

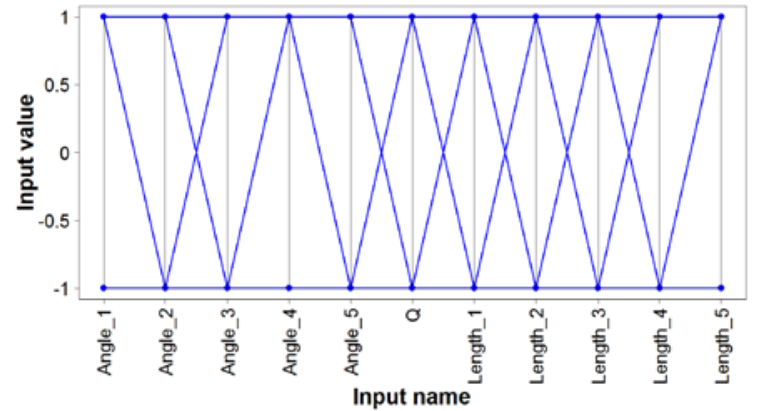

Figure 5. Added points with the maximal variance in $[-1,1]^{11}$ for $\Delta \mathrm{P}$.

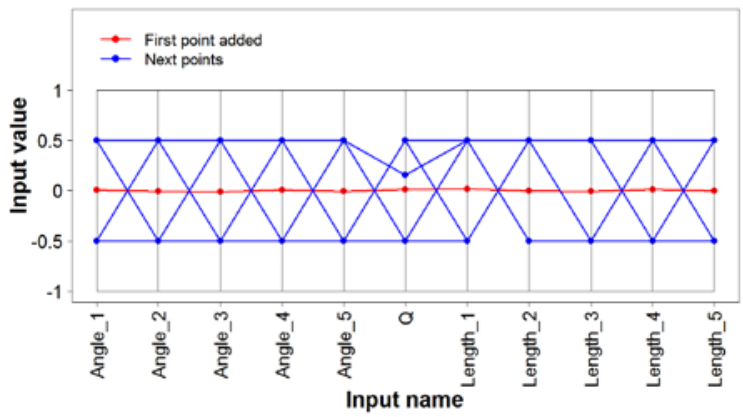

Figure 6. Added points with the maximal variance in $[-0.5,0.5]^{11}$ for $\Delta \mathrm{P}$.

To compare the effect of adding point either in $[-1,1]^{11}$ or in $[-0.5,0.5]^{11}, 10000$ points from a Sobol sequence were generated and used to compare the mean of their kriging variance. However the mean of the variance criterion was assessed by removing the central point of this sequence because it is the first added point of the second method. This mean criterion is computed on the $\Delta \mathrm{P}$ meta-model each time another point has been added at location of maximum kriging variance. The black curve (figure 7) shows the decrease of the mean variance when adding sequentially the points in $[-1,1]^{11}$ whereas the red curve is for $[-0.5,0.5]^{11}$. Initially, adding the central point is obviously worthy, and then the rate of improvement is rather equivalent.

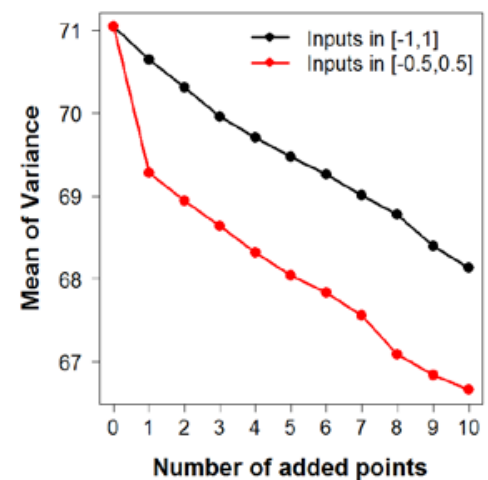

Figure 7. Comparison of the methods on the mean of variance criterion.

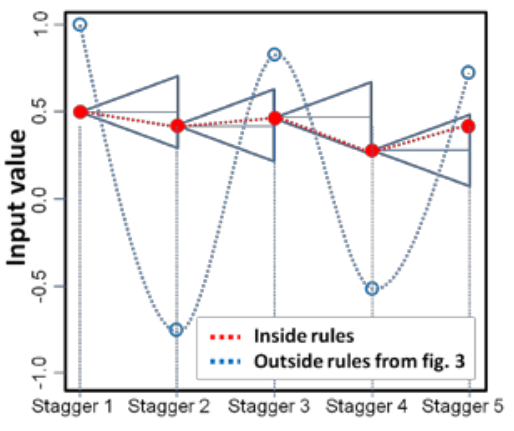

Figure 8: Examples satisfying the stagger angle constraints (red) or not satisfying them (blue, geometry from fig.3).

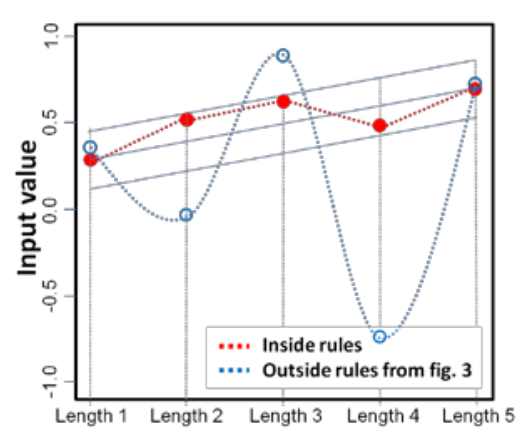

Figure 9: Examples satisfying the chord length constraints (red) or not satisfying them (blue, geometry from fig.3). 
The first method to be evaluated in this article will be the one that adds points of maximum kriging variance in the restricted domain $[-0.5,0.5]^{11}$.

\section{Adding geometrical constraints}

Points which are added must be relevant from a turbomachine point of view, in order to avoid strange geometries like in figure 3 . When adding geometrical constraints, the selection process for the additional points simply discards the geometries which are not considered credible, and iterates until a validated point is found. The rules are simply based on the observation that irrelevant geometries are characterized by abrupt variations and large amplitudes of both chord length and stagger angles. It does not make sense to have angles of incidence which vary greatly from one radius to another, and that the load is not distributed smoothly along the blade span. Two rules are therefore applied and must be verified simultaneously, one concerning stagger angles and one concerning chord lengths.

The rules are defined to limit variations from one radius to another. The examples of figures 8 and 9 show possible evolutions, when variation for stagger angles are limited to $+/-10 \%$, and chord lengths do not deviate from the mean line between bottom and top by more than $+/-10 \%$. For the sake of comparison, the variations of the fan of figure 3 are presented on the graph. It is obvious that these former geometries don't follow these rules.

The second method to be evaluated in this article will be the one that adds points of maximum kriging variance among the points that satisfy geometrical constraints.

\section{Adding a linear trend}

As introduced by the formula (7), the trend can be defined by the following formula (given in eleven dimensions): $m+\sum_{k=1}^{11} \propto_{k} h_{k}\left(x_{k}\right)$. This method can be used by considering the pressure rise variation $(\Delta \mathrm{P})$ which is rather linear, and systematically decreasing versus flow rate $(\mathrm{Qv})$. The linear trend will therefore have the following shape: $m+\alpha_{Q} Q$. In the article, the constant trend and the linear trend will be tested and compared for the ouput $\Delta P$.

\section{Comparing methods}

The two validation plans (in $[-1,1]^{11}$, and in $[-0.5,0.5]^{11}$ ) are used to assess a criterion based on the Root Mean Square Error (RMSE), according to the formula:

$$
R M S E=\sqrt{\frac{\sum_{i=1}^{N}\left(\boldsymbol{y}^{(i)}-\widehat{\boldsymbol{m}}\left(\boldsymbol{x}^{(i)}\right)\right)^{2}}{N}}
$$

$x^{(i)}$ is the $\mathrm{i}^{\text {th }}$ point of the test sample. $y^{(i)}$ is the true output value associated to $x^{(i)} . \widehat{m}\left(x^{(i)}\right)$ is the meta-model predicted output value associated to $x^{(i)} . N$ is the number of points in the test sample.

\section{Accuracy assessment in the Whole Domain}

The objective in this paragraph is to compare the quality of two different strategies of enrichment which use only 10 additional points each. Of course these points are not the same according to the strategy. The two methods will be compared to the so-called "initial" model, for which the training sample is limited to the initial sample of 127 points. In the first method, ten points of maximum variance have been selected in $[-0.5,0.5]^{11}$ and added to the initial training sample (forming a new training sample of 137 points). In the second model, ten points of maximum variance among points satisfying the geometrical rules have been added to the initial training 
sample (forming another training sample of 137 points). In addition with these sampling strategies, linear and constant trends on the pressure curves have been compared.

In summary, there are 3 models with constant trend for the pressure rise, three others with linear trend for the same output, and 3 models for the torque (only constant trend on torque). Table 1 gives the comparison for initial model and the 2 sampling methods.

It can be observed that the enrichment methods have a low incidence on the RMSE criterion since the mean error remains at $12 \mathrm{~Pa}$ for the pressure rise, and at $0.03 \mathrm{~N} . \mathrm{m}$ for the torque. Adding a trend is more promising since it improves the RMSE by 1 to $2 \mathrm{~Pa}$. .

\begin{tabular}{|l|c|c|c|}
\hline & \multicolumn{2}{|c|}{ RMSE on $\Delta \mathrm{P}$} & \multirow{2}{*}{ RMSE on T } \\
\cline { 1 - 3 } & $\begin{array}{c}\text { With } \\
\text { constant trend }\end{array}$ & $\begin{array}{c}\text { With linear } \\
\text { trend }\end{array}$ & \\
\cline { 1 - 3 } Initial & $12 \mathrm{~Pa}$ & $11 \mathrm{~Pa}$ & \multirow{2}{*}{0.03 N.m } \\
\hline Enrichment in [-0.5, 0.5] $]^{11}$ & $12 \mathrm{~Pa}$ & $10 \mathrm{~Pa}$ & 0.03 N.m \\
\hline Enrichment with geometrical rules & $12 \mathrm{~Pa}$ & $11 \mathrm{~Pa}$ & 0.03 N.m \\
\hline
\end{tabular}

Table 1: Error assessment of the meta-model in the whole domain

\section{Accuracy assessment in the Middle of the Domain}

Table 2 shows the results obtained with the same models than table 1 , but evaluated in the domain $[-0.5,0.5]^{11}$. Once again, the linear trend provides a rather good improvement of the RMSE of 1 to $3 \mathrm{~Pa}$. Comparing the two sampling methods, it shows this time a noticeable difference: the geometrical rules even deteriorate the meta-model globally. However it does not state if it is relevant for an optimization which is supposed to go only in areas of interest for turbomachine design.

\begin{tabular}{|l|c|c|c|}
\hline & \multicolumn{2}{|c|}{ RMSE on $\Delta \mathrm{P}$} & \multirow{2}{*}{ RMSE on T } \\
\cline { 1 - 3 } & $\begin{array}{c}\text { With } \\
\text { constant trend }\end{array}$ & $\begin{array}{c}\text { With linear } \\
\text { trend }\end{array}$ & \\
\cline { 1 - 3 } Initial & $10 \mathrm{~Pa}$ & $8 \mathrm{~Pa}$ & \multirow{2}{*}{0.05 N.m } \\
\hline Enrichment in [-0.5, 0.5] $]^{11}$ & $9 \mathrm{~Pa}$ & $8 \mathrm{~Pa}$ & 0.03 N.m \\
\hline Enrichment with geometrical rules & $19 \mathrm{~Pa}$ & $16 \mathrm{~Pa}$ & 0.06 N.m \\
\hline
\end{tabular}

Table 2: Error assessment of the meta-model in the middle of the domain 


\section{OPTIMIZATION}

The meta-models are then compared on two optimization problems:

- Maximize $\Delta \mathrm{P}, \mathrm{T}=<1 \mathrm{~N} . \mathrm{m}, \mathrm{Q}$ set at a given nominal flow rate

- Minimize $\mathrm{T}, \Delta \mathrm{P}>=250 \mathrm{~Pa}, \mathrm{Q}$ set at a given nominal flow rate

Figures 10 and 11 show the set of data for the optimized solutions (maximizing $\Delta \mathrm{P}$ ), using kriging respectively without constant or linear trend. In both cases, the first two models are rather similar, i.e. with the initial DoE and the enrichment in the middle. At this point, it would indicate that the meta-model is already relevant and a small enrichment, despite being based on a statistical approach with variance, does not improve the result. In addition, the small improvement noticed on the RMSE with the trend does not really change the optimum proposed. For information, a view of an optimized fan is shown in figure 12.

The result is slightly different when using the meta-model which has been enriched with points fulfilling some criteria based on design rules. The set of data shows smaller variations in stagger angles and in chord length from a radius to another, indicating that the rules have modified the meta-model around their domains of validity. However, despite being less chaotic, the stagger angles still show a big variation from radius 1 to 2 and from radius 4 to 5 . For the chord length, there is still a big change from radius 4 to 5 . As a consequence, it must be noticed that the optimized solutions using the meta-model with rules are outside these rules.

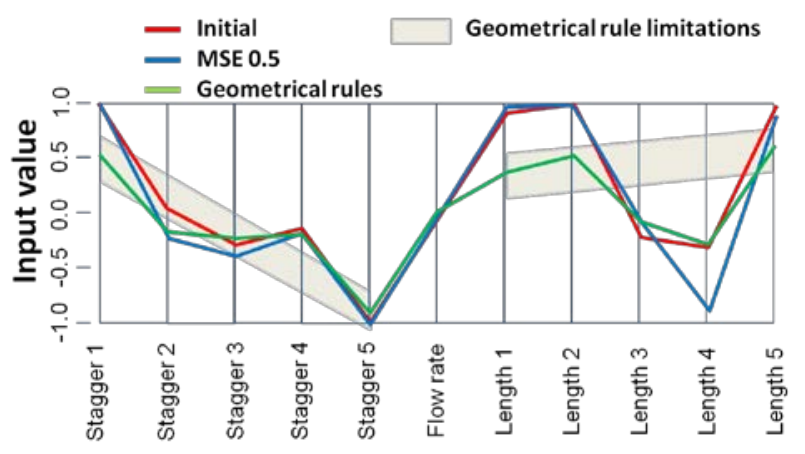

Figure 10: solutions when using $\Delta \mathrm{P}$ metamodels with constant trend (first optim.)

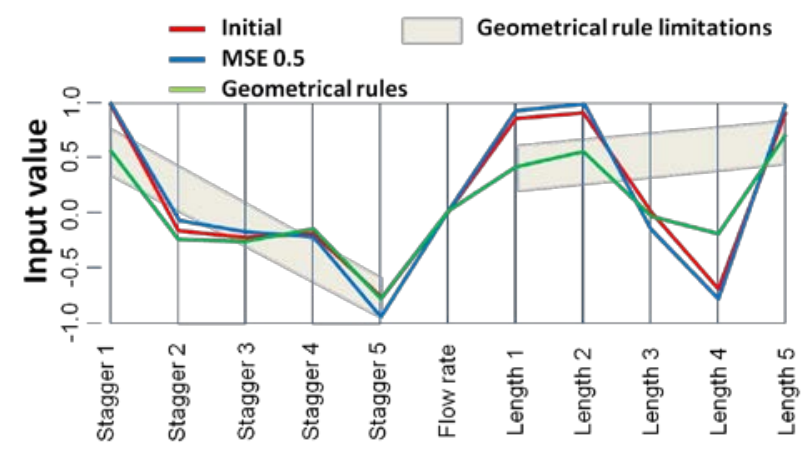

Figure 11: solutions when using $\Delta \mathrm{P}$ metamodels with linear trend (first optim.)

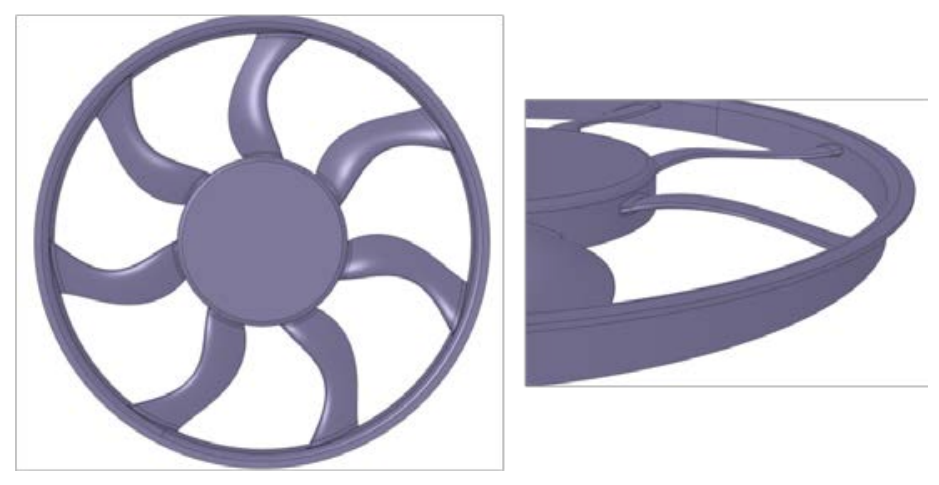

Figure 12: optimized fan

Errors given by the meta-models are presented in table 3, where the actual performances are those given by the simulation. The table shows the results obtained when using the linear trend in 
the meta-model, however the results are quite similar without it as demonstrated by comparing figures 10 and 11. All proposed solutions are within a reasonable range of performance which is at the state of the art for such fans, i.e. close to $50 \%$ of static to static efficiency.

The enrichment in $[-0.5,0.5]^{11}$, despite showing no RMSE improvement, has however brought a noticeable improvement in efficiency $(+0.4 \%$ and $+1.5 \%)$ versus the initial DoE. As no improvement is noticed in accuracy between these optimizations from the two meta-model, it would suggest that expecting accuracy is not the point, but rather having the good trend.

The last model which has introduced some rules for the choice of added points is finally the less performing one. Efficiencies are almost 3\% below those obtained with the enrichment in the middle. Removing some points where the variance was important because of the geometrical criteria has probably lowered the ability of the kriging process to find good trends in the whole domain.

\begin{tabular}{|c|c|c|c|c|}
\hline & \multicolumn{2}{|c|}{ Optim $1($ Maximize $\Delta \mathrm{P})$} & \multicolumn{2}{|c|}{ Optim 2 (Minimize T) } \\
\hline & $\begin{array}{l}\text { Values with linear } \\
\text { trend }\end{array}$ & $\begin{array}{l}\text { Errors with linear } \\
\text { trend }\end{array}$ & $\begin{array}{l}\text { Values with linear } \\
\text { trend }\end{array}$ & $\begin{array}{l}\text { Errors with linear } \\
\text { trend }\end{array}$ \\
\hline Initial & $\begin{array}{l}\Delta P=186 \mathrm{~Pa} \\
T=1.07 \mathrm{~N} . \mathrm{m} \\
E=\mathbf{4 7 . 9} \%\end{array}$ & $\begin{array}{l}\Delta(\Delta P)=11 \mathrm{~Pa} \\
\Delta(T)=0.07 \mathrm{~N} . \mathrm{m} \\
\Delta(E)=\mathbf{6 . 4} \%\end{array}$ & $\begin{array}{l}\Delta P=226 \mathrm{~Pa} \\
T=1.29 \mathrm{~N} . \mathrm{m} \\
E=48.5 \%\end{array}$ & $\begin{array}{l}\Delta(\Delta P)=24 \mathrm{~Pa} \\
\Delta(T)=0.06 \mathrm{~N} . \mathrm{m} \\
\Delta(E)=7.8 \%\end{array}$ \\
\hline $\begin{array}{l}\text { Enrichment } \\
\text { in } \\
{[-0.5,0.5]^{11}}\end{array}$ & $\begin{array}{l}\Delta P=190 \mathrm{~Pa} \\
T=1.08 \mathrm{~N} . \mathrm{m} \\
E=\mathbf{4 8 . 3} \%\end{array}$ & $\begin{array}{l}\Delta(\Delta P)=11 \mathrm{~Pa} \\
\Delta(T)=0.08 \mathrm{~N} . \mathrm{m} \\
\Delta(E)=7.3 \%\end{array}$ & $\begin{array}{l}\Delta P=235 \mathrm{~Pa} \\
T=1.3 \mathrm{~N} . \mathrm{m} \\
E=\mathbf{5 0} \%\end{array}$ & $\begin{array}{l}\Delta(\Delta P)=15 \mathrm{~Pa} \\
\Delta(T)=0.11 \mathrm{~N} . \mathrm{m} \\
\Delta(E)=7.8 \%\end{array}$ \\
\hline $\begin{array}{l}\text { Enrichment } \\
\text { with rules }\end{array}$ & $\begin{array}{l}\Delta P=173 \mathrm{~Pa} \\
T=1.06 \mathrm{~N} . \mathrm{m} \\
E=45.1 \%\end{array}$ & $\begin{array}{l}\Delta(\Delta P)=27 \mathrm{~Pa} \\
\Delta(T)=0.06 \mathrm{~N} . \mathrm{m} \\
\Delta(E)=10.0 \%\end{array}$ & $\begin{array}{l}\Delta P=218 \mathrm{~Pa} \\
T=1.28 \mathrm{~N} . \mathrm{m} \\
E=47.1 \%\end{array}$ & $\begin{array}{l}\Delta(\Delta P)=32 \mathrm{~Pa} \\
\Delta(T)=0.04 \mathrm{~N} . \mathrm{m} \\
\Delta(E)=8.4 \%\end{array}$ \\
\hline
\end{tabular}

Table 3: Actual performances for the optima, and errors of meta-model predictions

\section{CONCLUSIONS}

Investigations have been conducted in order to define the most relevant strategy for metamodeling with sequential enrichment. It is assumed that the number of added points must be limited, and therefore a smart solution for optimizing the proposed runs must be found.

Two enrichment methods have been proposed, one being based on the variance in the center of the domain, and one adding some geometrical rules aimed to discard non conventional designs. In addition, a linear trend on the pressure rise has been included in the models and compared to the usual constant trend.

Accuracy assessment obtained with a RMSE criterion on validation designs has shown that limiting the sampling in the center of the domain seems to be an interesting strategy for scarce additional points, and that adding the trend improves the models. This would be all the more to be confirmed since tests with optimization have shown a small gain on maximum efficiency. 
Conversely using geometrical rules for the enrichment does not help and even deteriorates the quality of both the meta-model and the optimization. It is still not explained, but presuming solutions is ultimately more counterproductive than letting the model learn from all points, including from those that are not good geometries.

\section{ACKNOWLEDGMENTS}

This work is being supported by the French Research Agency ANR [12], for the project PEPITO (“Plan d’Experience Pour l’Industrie du Transport et l’Optimisation”)

\section{REFERENCES}

[1] K. Bamberger, T. Carolus, “Optimization of axial fans with highly swept blades with respect to losses and noise reduction”, FAN 2012 Senlis (France) 18-20 April 2012

[2] T. Verstraete, P. Roytta, M. Henner, et al. "Design and off-design optimization of a fan for automotive applications". In: Proceedings of the 9th European conference on turbomachinery fluid dynamics and thermodynamics (ETC’9), Istanbul, Turkey, 2011, pp. 1485-1496.

[3] Henner M., Demory B., Franquelin F. and al., (2014). "Test rig effect on performance measurement for low loaded high diameter fan for automotive application”. ASME Turbo Expo, Frankfort, Germany, 2014.

[4] Qian P. Z., (2009). “Nested latin hypercube designs”. Biometrika.

[5] Rasmussen C. E. and Williams C. K. I., (2006). "Gaussian Processes for Machine Learning”. MIT Press.

[6] Loic Le Gratiet. "Multi-fidelity Gaussian process regression for computer experiments”. Autres [stat.ML]. Université Paris-Diderot - Paris VII, 2013. Français. 〈tel-00866770v2〉.

[7] Ribaud M., Blanchet C., Gillot F., and Helbert C., (2018). "Robustness kriging-based optimization”. https://hal.archives-ouvertes.fr, <hal-01829889>

[8] Z. Zhang, B. Demory, M. Henner, B. demory, F. Franquelin, "Space infill study of Kriging meta-model for multi-objective optimization of an engine cooling fan", ASME Turbo Expo 2014, Dusseldorf, GT2014 June 16-20.

[9] L. Soulat, P. Ferrand, S. Moreau, S. Aubert, M. Buisson, "Efficient optimisation procedure for design problems in fluid mechanics”, Computers \& Fluids, Volume 82, 15 August 2013, Pages 7386

[10] M. Buisson, P. Ferrand, L. Soulat, S. Aubert, S. Moreau, C. Rambeau, M. Henner, ”Optimal design of an automotive fan using the Turb-Opty meta-model”, Computers \& Fluids, Volume 80, 10 July 2013, pp 207-213 
[11] Z. Zhang, M. Buisson, P. Ferrand, M. Henner and F. Gillot, "Meta-model based optimization of a large diameter semi-radial conical hub engine cooling fan”, Mechanics \& Industry, Volume 16, Number 1, 2015.

[12]http://www.agence-nationale-recherche.fr/projetanr/?tx_lwmsuivibilan_pi2[CODE]=ANR-14CE23-0011 